\title{
Redes de Comunicación en Smart Grid
}

\author{
Esteban Mauricio Inga Ortega ${ }^{1}$
}

\section{Resumen}

Las Redes de Comunicación sobre Smart Grid, en conjunto con una metodología de gestión apropiada, pueden aportar opciones que busquen ofertar servicios adicionales a los consumidores del suministro eléctrico, en ese aspecto el presente documento hace referencia a la importancia del consumidor, es decir al cliente mismo del suministro eléctrico, dentro de la propuesta de una Red Eléctrica Inteligente. Ante esto se advierte la necesidad de buscar la mayor eficiencia y fiabilidad de la red eléctrica en general y existen propuestas para lograrlo, ya sea en la etapa de generación, de transmisión o de distribución; pero la mayoría de los proyectos iniciales se concentran en la etapa de distribución, lugar donde se generan diferentes tipos de pérdidas de energía. Los proyectos iniciales se implementan también para disminuir el porcentaje de apagones, por lo que se ha trabajado en la automatización de las sub-estaciones colocando equipos de última tecnología como IED's - Dispositivos Electrónicos Inteligentes para los sistemas de protecciones, que en conjunto con el empleo de protocolos de comunicación, son aplicados para realizar cortes y reconexiones ágiles y confiables del suministro eléctrico. La etapa que se conoce como AMI (Infraestructura de Medida Avanzada), donde los diferentes estudios e investigaciones han centrado su interés ya sea por las entidades gubernamentales o privadas, dejan en segundo plano estudios para mejorar o dotar de servicios adicionales a la etapa final o cliente.

Palabras clave: Smart Grid, Smart Metering (Medición inteligente), HAN (Red de área doméstica), Eficiencia Energética, Calidad de la Energía.

\begin{abstract}
A Communication Networks based upon Smart Grid, in conjunction with an appropriate management methodology, could contribute to options that look into the provision of additional services to consumers of electricity. In this respect, the following paper makes reference to the importance of the consumer as a client in itself, in regards to electrical supply within the proposal of a grid. In view of this, it is paramount to search for the best efficiency and reliability of the electrical network in general- and proposals already exist to do so, whether it be at the stage of generation, transmission or distribution. However, the initial projects in the majority of cases only concentrate on the distribution stage, where many types of energy losses are generated. The initial projects are implemented as well to reduce the percentage of power cuts, that is why work has been done on the automation of substations placing equipment of the latest technology such as IED's - Intelligent Electronic Devices for protective systems; along with the application of communication protocols which are applied to power cuts, to enable fast and reliable reconnections of power supply. The stage that is known as AMI (Advanced Measurement Infrastructure), where research and different studies have mostly focused their attention - be it due to governmental or private entities - disregards the improvement and provision of additional services to the final stage - which is the customer.
\end{abstract}

Keywords: Smart Grid, Smart Metering, HAN (Home Area Network), Eficient Energy, Power Quality.

\footnotetext{
${ }^{1}$ Ingeniero Eléctrico, Master en Educación, Director de carrera de Ingeniería Electrónica de la Universidad Politécnica Salesiana, sede Quito, Estudiante de Doctorado en Ingeniería de Proyectos de la Universidad Tecnológica de Panama. Autor para correspondencia: einga@ups.edu.ec
}

Recibido: 15 - Marzo - 2012; Aprobado tras revisión: 22 - Mayo - 2012

Forma sugerida de citación: Inga E. (2012). "Redes de Comunicación en Smart Grid". INGENIUS. N${ }^{\circ}$, (enero/junio). pp 36-55. ISSN: 1390-650X 


\section{Introducción}

En la actualidad varias empresas de generación y distribución se preguntan cómo lograr una mayor eficiencia para el consumo de la energía, reducir las pérdidas que por diferentes causas provocan sustanciales desniveles económicos, aumentan la demanda de potencia, con el consecuente impacto ambiental que esto acarrea. En distribución, el objetivo de la reducción de pérdidas, se ejecuta mediante una gestión autónoma que identifique y controle el verdadero consumo. La eficiencia sobre el consumo permite realizar un análisis del mismo y así entregar opciones de gestión que coadyuvan y posibilitan la reducción del consumo, sin olvidar la optimización en la infraestructura de la red. Es decir, en conjunto ofrecen un mejor servicio al cliente otorgando lo que ellos más requieren, como son, ventajas comerciales, tarifas reducidas, control y administración local de su verdadero estado.

En el ámbito del usuario se puede evitar lectura manual, y permitir la eliminación de recibos por lecturas. La tarificación flexible es una oportunidad que brinda una red inteligente para optimizar el consumo de la energía.

Al mismo tiempo un Smart Grid pretende que la operación en los sistemas tradicionales de producción, distribución, generación y consumo energético reduzcan las interrupciones en el servicio, las mismas que al ser representadas en costes resultan significativos por las diferentes actividades que dependen de este insumo.

Con el aporte de varios campos científicos y tecnológicos, la red eléctrica se puede transformar en una red de alta interconectividad, en la que converjan las tecnologías de la información [1], las redes de comunicación industrial, los sistemas inteligentes de medición, las fuentes de energía renovable y la generación distribuida. Este artículo pretende describir las características principales de una red inteligente, los logros alcanzados y los retos que motiven a la investigación y desarrollo científico en nuestro país dentro de ese campo.

Con estos antecedentes podemos centrar nuestro aporte en el cliente-consumidor, quien se ve limitado a realizar el pago de su planilla sin disponer de una oferta de mayores servicios que el suministro eléctrico. En un Smart Grid se plantea que la carga refleje también la mejora y la eficiencia energética. En muchas propuestas se compara al Smart Grid con el Internet por ser gestionado en dos vías, dado que circula la información de forma bidireccional, y porque está conformado por diferentes nodos dispersos que se gestionan de manera eficiente a lo largo de todo el sistema.

Se plantea que un Smart Grid además dote al cliente de otros servicios como el control de electrodomésticos, ahorro de energía [2], reducción de costes por el consumo del suministro eléctrico, para que el cliente vea de forma transparente el incremento de la fiabilidad en la lectura del suministro, que pueda optar por prepago del suministro eléctrico según lo que el usuario crea necesario. En resumen, facilitar al cliente herramientas que le ayuden a decidir cómo y cuándo consumir de forma responsable el suministro eléctrico. Es importante entonces realizar estudios en Ingeniería de Proyectos que, mediante una metodología de gestión [3], analicen un modelo nuevo de red de comunicación en busca de brindar servicios adicionales al cliente, ya sea en hogares, edificios [4] o industrias. Al incluir al cliente como parte activa del sistema eléctrico, según Smart Grid, se pretende ser constante en la petición diaria del suministro eléctrico y con esto reducir los picos de la demanda. 
En el aspecto de atención por demanda se debe tener en cuenta los siguientes aspectos:

- La disponibilidad de sistemas avanzados que permitan gestionar la demanda.

- Por parte del operador: acción comercial segmentada según perfiles de uso individuales y calidad del servicio.

- Por parte del usuario: concienciación energética y optimización del patrón de consumo.

- La proliferación de dispositivos que incorporen o permitan una gestión inteligente del consumo de energía y que tengan impacto en la generación, teniendo en cuenta los siguientes factores:

Uso en períodos de baja tarifa.

Modos económicos de funcionamiento.

Respuesta a nuevos usos (p.ej. autos eléctricos)

Soporta nuevos modelos de gestión.

\section{Generalidades}

\subsection{Infraestructura en general}

Las redes eléctricas inteligentes podrán cambiar su diseño y topología de acuerdo a los avances tecnológicos, pero es importante conocer las características básicas de una REI (Red Eléctrica Inteligente - Smart Grid) [5].

\subsection{GIS (Sistema de información geográfica) [7]}

La calidad de los datos que existe en el GIS debe ser excepcional dado que no es aceptable tener datos incorrectos en un sistema que controla automáticamente el sistema de distribución eléctrica. Los errores pueden ocasionar

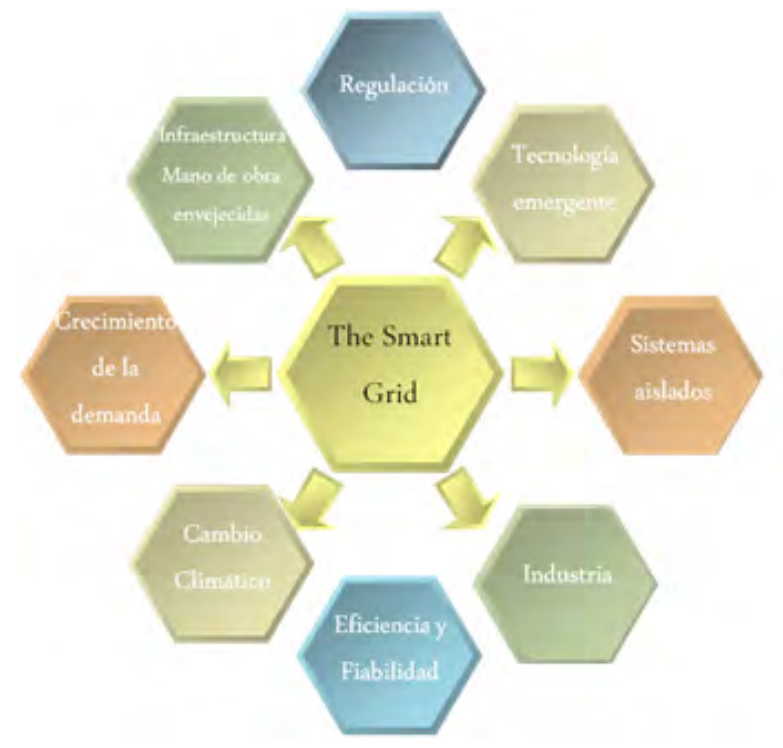

Figura 1. Detalle de las Redes Eléctricas Inteligentes. [6]

cortes de mayor tiempo o pero aún accidentes. Las necesidades de datos para las operaciones de Smart Grid sugieren que se debe medir el tiempo que toma a un cambio en reflejarse en el GIS puesto que a medida que el tiempo aumenta también lo hace el riego que algo salga mal. Es necesario entonces que el GIS tenga actualizada la base de datos con el objeto de tener una correlación exacta entre las instalaciones del cliente y el sistema eléctrico por lo cual el GIS debería ser casi perfecto de todo el sistema eléctrico.

\subsection{AMI (Infraestructura de medición avanzada)}

En el diseño de una red inteligente se prevé el uso de medidores digitales avanzados con dos vías de comunicación [8] que tengan la capacidad de conectar y desconectar servicios a distancia, registrar formas de onda, vigilar la tensión y la corriente. Estos deberán sustituir a los medidores actuales en el mismo lugar para no modificar el diseño en grandes dimensiones. Este tipo de medidores pueden tener datos a disposición de los centros de 
operación y planificación con la finalidad de lograr una mayor fiabilidad y utilización de recursos (Líneas, transformadores). De igual manera ofrecen lecturas de tiempo real, dando la posibilidad de equiparar los patrones de distribución de carga en el sistema.

\subsection{OMS (Sistema de gestión de cortes)}

En esta etapa de un Smart Grid se pretende identificar y restaurar cortes de energía de manera ágil y eficiente. Un OMS puede servir no solamente como una herramienta para los servicios públicos del departamento de operaciones, sino como una herramienta de servicio al cliente, de planificación, de gestión de activos, una herramienta a través de la cual los departamentos de ingeniería y asuntos regulatorios pueden encontrar información recopilada en su base de datos.

\subsection{SCADA (Sistema de adquisición, supervisión y control de datos)}

En este concepto se puede advertir la existencia de un HMI (Human Machine Interface Interfaz Hombre Máquina) bajo un software diseñado e instalado sobre un servidor para el control de producción, mismo que puede dotar de comunicación con dispositivos de campo (autómatas), controlando el proceso de forma automática desde la pantalla de monitorización. Este sistema provee de toda la información que se genera en el proceso productivo a diversos usuarios, esto se pueda dotar de información a diferentes niveles del sistema como supervisión, control de calidad, control de producción, almacenamiento de datos. En resumen el SCADA contiene diversas soluciones para captura de información de un proceso o planta no necesariamente industrial lo que permite realizar análisis en tiempo real o estudios posteriores y así obtener indicadores que permitan retroalimentar sobre un proceso u operación.

\subsection{DMS (Sistema de distribución de gestión)}

La plataforma de gestión energética integrada se desarrollará, con funciones avanzadas para hogares, es decir la gestión de la energía por los consumidores y así mejorar las operaciones de los sistemas de distribución por sus utilitarios. Esta plataforma se integrará al AMI (Advanced Metering Infrastructure) como un portal a un hogar, para que entregue la respuesta de la demanda, la automatización del hogar para la conservación de la energía, óptimo despacho de la generación distribuida, el almacenamiento, carga en el sistema de distribución, y los controles para que el sistema de distribución de una entidad de despacho permita colaborar con otras entidades en la carga de la red.

La gestión de energía de este tipo permitirá a los consumidores entre otros servicios tomar el control, automatización de la energía, prácticas de conservación y respuesta a la demanda sobre la base de sus preferencias personales.

La domótica por ejemplo se basa en Smart Meter y productos ecoDashboard de General Electric. El Smart Meter puede usar una red ZigBee que se encargará de comunicar a los aparatos domésticos, y el tablero de instrumentos tendrá automatizados los controles de sus operaciones. Además, esta plataforma ofrecerá servicios auxiliares para el servicio público local como, rotación de la reserva, la regulación de carga (load-following regulation), e intermitencia por la gestión de la energía eólica y solar. 


\subsection{DA (Automatización de distribu- ción)}

Esta etapa se encarga de la supervisión, el control y las funciones de comunicación situados en el alimentador, dentro del diseño se encuentra en las áreas de protección y de conmutación (suelen estar en el mismo dispositi$\mathrm{vo)}$.

Los dispositivos de ésta etapa pueden interrumpir la corriente de falla, monitorear las corrientes y voltajes, comunicarse con otros dispositivos similares y ser auto configurables, el sistema tiene la finalidad de restaurar el servicio a los clientes, la capacidad en flexibilidad y rapidez para reconfigurar la red de alimentadores, adicionalmente se requiere que la distribución de los componentes tenga la capacidad suficiente para aceptar la transferencia y además requiere del sistema de protección para aislar la falla en la topología reconectada. Es decir que una red eléctrica inteligente [9] a más de conectar a los clientes de una subestación con bajo costo permite una alimentación rápida y flexiblemente reconfigurada.

Entonces en el futuro se puede diseñar sistemas de distribución en una red con conexión a múltiples etapas de distribución, la misma que estaría conectada a múltiples subestaciones, el punto importante está en los alimentadores de la red que permita un sistema interconectado de alimentadores. Puede ser un inconveniente si en el sistema de distribución actual se coordina los dispositivos de protección ya que en un smart grid la topología permitida es flexible, es decir se tendrá que planificar adecuadamente para lograr una coordinación correcta para una variedad de configuraciones posibles.

\section{Smart grid}

Generar una metodología de trabajo sobre la base de los conceptos teóricos ya conocidos para facilitar el incremento de la eficiencia energética así como su confiabilidad, ofertados y centralizados en el cliente, es lo que se necesita investigar en la actualidad. Las motivaciones para optar por un proyecto de este tipo será el interés sobre la etapa más importante de un Smart Grid que es el consumidor final y de la misma manera que una operadora de telefonía celular oferta servicios pos-venta, hacerlo con el suministro eléctrico, de tal manera que se genere un Smart Grid pensando también en cliente-consumidor.

Sin duda alguna que el impacto social de estas soluciones genera expectativas positivas en gran magnitud para las empresas de distribución y su relevancia estará justificada cuando se pretenda eliminar los diferentes subsidios al suministro eléctrico, como sucede en este momento en nuestro país Ecuador. Los datos serán analizados desde la perspectiva de las facilidades que puedan brindar las diferentes arquitecturas de los protocolos de comunicación y los conceptos para formar redes de área para hogares, edificios e industria, así como el análisis de la velocidad, cobertura, adaptabilidad, compatibilidad con el suministro eléctrico.

El análisis de las diferentes arquitecturas de los protocolos de comunicación planteados para la implementación en Smart Grid debe buscar el diseño de una nueva red de comunicación que colabore a gestar servicios adicionales al cliente.

Si en estos momentos se decidiera eliminar al subsidio simplemente como política de estado, esto solo se vería reflejado en el valor de 
la planilla, sin tener servicios adicionales que por lo menos coadyuven al cliente a ser consciente que recibe un valor agregado de parte de la empresa distribuidora, es decir un mejor servicio que además le ayude a ser eficiente con su consumo u ofertas adicionales que el estado local le entregue como confiabilidad del sistema.

Es importante introducir al cliente completamente en el modelo del Smart Grid para que la solución sea bidireccional. Entre los resultados se espera que los diferentes modelos de comunicación se adhieran al modelo Smart Grid, con la finalidad de ofertar servicios adicionales a los clientes del suministro eléctrico, estos resultados se podrán adjuntar a las propuestas de los mapas de ruta que se los utiliza para implementar Smart Grid, el modelo de red podrá acompañar a la etapa AMI (Infraestructura de Medida Avanzada) sobre una red eléctrica inteligente para la resolución de las diferentes necesidades urgentes de la generación eléctrica.

El nuevo concepto de la Ingeniería Eléctrica considera que se necesita de las TIC para avanzar en el proceso que busca eficiencia y confiabilidad de la energía eléctrica, esto ayudará en parte a disminuir el impacto ambiental y la reducción CO2, por lo que se analiza la posibilidad de enlazar nuevas competencias al perfil de los profesionales asociados y que se desprenden de los procesos de generación, transmisión, distribución y consumo del recurso eléctrico, al mismo tiempo la inclusión de energías limpias como solar y eólica que necesitan tener la opción de comunicación en dos vías, es decir se sigue manejando el concepto de bidireccionalidad de nuestra red ejemplo como lo es "el internet".

De esta forma se puede distribuir desde la carga otro tipo de generación eléctrica, como fuentes limpias dentro del modelo de Smart Grid permitiendo tener un concepto circular que retorna a las diferentes etapas antes mencionadas que el cliente generó y mediante políticas de estado poder incentivar este tipo de generación, por ejemplo: otorgando un coste superior por producir energías limpias de forma doméstica (hogar-edificio-industria), el usuario puede desvincular de su planilla de consumo el valor de generación y así solo pagar el excedente por el consumo de la red eléctrica convencional, esto facilita control, monitoreo, re-distribución del recurso energético limpio, de igual manera se debe tomar en cuenta que no todas las redes existentes de comunicación tienen los mismos requerimientos técnicos y que no todas las tecnologías son apropiadas para todos los usos, razón importante para el respectivo análisis e investigación.

Las redes eléctricas inteligentes pueden denotar la formación o coexistencia de dos redes a través de su automatización e interconexión en la que los productores o suministradores de energía y los consumidores se interconectan entre dos redes, es decir se implementa una red bidireccional de información análoga a la de la electricidad, ofreciendo información integra con la finalidad de optimizar y agilitar la toma de decisiones en los procesos de gestión de la red eléctrica.

Dentro de los cuestionamientos para hacer eficiente el suministro eléctrico podemos citar los siguientes:

- Cambio Climático

- El coste de combustibles fósiles usados para satisfacer la demanda

- Pérdidas en la red eléctrica

A más de estos puntos tratados desde un 
Smart Grid se adhieren las necesidades de impacto económico que generan los cortes de energía por la falta de fiabilidad, dichos apagones no son muchas veces identificados hasta que el usuario levanta un reclamo y a nivel mundial usuarios que generan servicios a múltiples clientes entre los que están;

- Comunicaciones Móviles

- Compra y venta telefónica

- Reservaciones de servicios aeroportuarios

- Manufactura

- Operaciones bancarias en línea

- Transacciones varias

Impactos que pueden ser mitigados desde la perspectiva de una red eléctrica inteligente, sin descartar que por cada kilovatio se requiere en algunos países se quema más carbón y petróleo aumentando la emisión de los gases de efecto invernadero, pero todos los desperfectos de la red en su mayoría repercute de forma directa en la planilla que paga el consumidor final. La planilla del consumidor se ve afectada cuando existen los famosos picos de demanda, para esto actúan plantas emergentes con el fin de satisfacer la demanda, plantas que muchas de ellas usan combustible fósiles.

Una de la etapas del Smart Grid que se plantea como AMI, tiene la capacidad de detectar problemas en el sistema, permitiendo gestionar la demanda y por ende una operación más eficiente pero desde el punto de vista de las empresas distribuidoras, estos aspectos resultan importantes ya que proporcionaran información casi en tiempo real al consumidor y le permitirán planificar el consumo ya sea por el usuario o por la empresa eléctrica de distribución.
Es entonces importante por ejemplo conocer de parte del cliente en tiempo real el coste de la energía para que en los tiempos de mayor demanda se pueda elegir de parte del consumidor si se debe acceder a tal o cual equipo / electrodoméstico, esto permitiría una reducción en la planilla si existe planificación responsable.

Los primeros bocetos del Smart Grid plantean adicional al modelo inicial a las microredes de fuentes de energía limpia, las mismas que se sitúan de forma alejada a las diferentes subestaciones tradicionales, pero es importante que se las gestione desde el mismo modelo ya que en muchos casos puede ser generada desde el consumidor final del suministro eléctrico, aunque pueden existir plantas que pertenecen al sector público-gubernamental. La inclusión de las energías limpias dependen de la situación climatológica [10] y si no se tiene control en aspectos como de donde se genera, cuando y cuanto lo genera, es decir si no existe comunicación con el ente regulatorio sería un trabajo infructuoso y costo por mantenimiento, gestión y control.

Un modelo de red de comunicación que permita gestionar estos consumos y el resto de generadores de forma eficiente, es lo que se necesita, convirtiendo en este caso al usuario tradicional en fuente y suministro de energía.

Entonces lo importante es satisfacer las necesidades de los clientes a más de dotar de servicios pos-venta, todo esto enmarcado desde los conceptos y metodologías para ser implementados por empresas distribuidoras, realidad propia de Ecuador, para esto se deberá asumir los cambios y el crecimiento de la ciencia y la tecnología como son las TIC, las mismas que serán aplicadas de forma integral y global al problema mencionado. 
Existe impacto social sin duda alguna ya que dentro de los diferentes foros, avances y pruebas de Smart Grid se advierte que podría ser invasiva al plantear que se requiere de una conexión directa con todos los clientes finales para lograr comprobar el estado de sus líneas, consumos y suministros, con el fin de actuar antes del aviso de los fallos.

Además es importante analizar desde el punto de vista de la empresas distribuidoras ya que su negocio es vender el recurso energético y al ser más eficiente el sistema, podría disminuir su venta, por tal razón se ofertaría un nuevo plan de negocio que involucra servicios adicionales denominados pos-venta y bajo el análisis de los estándares de comunicación lograr la interoperabilidad con electrodomésticos, equipos de calefacción, aire y los futuros coches eléctricos, etc. A esto no se debe descartar reformas y normativas legales para incentivar la eficiencia y regeneración de la infraestructura de la red eléctrica.

En los actuales momentos las aplicaciones en Ecuador en relación a las diferentes etapas de automatización avanzan paulatinamente de acuerdo a las necesidades del mapa de ruta por intentar llegar a un Smart Grid, las etapas más elaboradas son GIS y AMI, siempre se maneja desde la etapa de distribución que por años ha sido el punto donde existen mayores dificultades. Ha existido a más de estos esfuerzos, propuesta de los entes regulatorios como el CONELEC que plantea reformas y normativas para generar la bidireccionalidad en el caso de que un usuario genere energía limpia y puede revertir el excedente del mismo. Otro avance lo genera el Centro Nacional de Control de Energía que tiene en estos momentos su esfuerzo centrado a la implementación de PMU's (Sincronofasores) para indicar la tensión y corriente en los tramos necesarios con un muestreo de 30 veces por segundo aproximadamente y aportarán con información acerca del régimen permanente, estos equipos aportarán datos del régimen transitorio de la red eléctrica, como sobrecargas para tomar decisiones con un tiempo prudencial y así evitar apagones.

Todas son diferentes gestiones encaminadas a buscar confiabilidad en el sistema, a esto se adicionan los servicios que concienticen al usuario a ser eficiente con su consumo, a estar contento por el servicio que recibe, ofertas, prepago, incentivos por generar energía limpia, es decir un pliego de ofertas acorde a la tecnología existente.

En estos momentos Ecuador es un país que ha eliminado el subsidio al gas licuado de petróleo para uso en edificios residenciales que usan un sistemas denominado gas centralizado, poco seguro y confiable, impacto a la salud por los diferentes efecto tóxicos de sus diferentes equipos que lo usan, esta situación tiene impacto social en las planillas mensuales por el uso del gas, y si a esto se suma los costos de energía eléctrica, agua, teléfono fijo y móvil el coste aumenta. Una alternativa es implementar energías limpias en estas edificaciones en lugar del uso del gas, y en muchos lugares se podría vender el excedente de energía pero la interrogante es cómo se monitorea, gestiona y automatiza, o cómo se reduce de la planilla el costo por generación doméstica de energía, la respuesta es que sin el uso de las comunicaciones no se podría lograr.

Además con una metodología de gestión de proyectos [11] de redes de comunicación pensado en el cliente ya sea que esté en hogares, edificaciones e industrias, dotando de servicios adicionales al recurso de la energía eléctrica que brinda una empresa distribuidora, el mo- 
delo podrá satisfacer a ambos puntos, tanto a la etapa de distribución como al cliente denominado abonado, estos nuevos conocimientos deberán satisfacer las necesidades y generar nuevas investigaciones a la comunidad científica, este proyecto apoyará al modelo teórico más grande que es Smart Grid, el grado de pertinencia pensado en el cliente fundamenta la necesidad del proyecto encaminado a mejorar no solo el modelo de eficiencia y confiabilidad energética sino a la comunidad con un grado significativo de responsabilidad sobre el impacto ambiental.

Esta estrategia puede ser la implantación o adopción de una metodología apropiada o el desarrollo de una nueva, de acuerdo al modelo de Smart Grid, los objetivos están centrados en el desarrollo de dicha estrategia, así como el plan de implantación mediante un proyecto piloto que colabore a medir el éxito de la estrategia aplicada, el proceso de planificación del proyecto deberá entonces ser flexible, continuo y con retroalimentación en cada una de sus etapas.

La visión general del modelo Smart Grid es obtener una red eléctrica auto gestionable y equilibrada [12], la misma que puede admitir cualquier fuente de energía como; carbón, sol, viento y que transforma esta energía para el uso de los consumidores en aplicaciones tales como calor, luz, agua caliente; todo esto con la mínima inversión del hombre permitiendo a la sociedad optimizar el uso de las diversas fuentes de energía renovable y contribuir en la reducción del impacto ambiental. Además dicha red eléctrica inteligente permite la comunicación en tiempo real entre el consumidor y las empresas eléctricas, optimizar la energía consumida según criterios ambientales y desde el punto de vista de su respectivo coste.
La transición que ha generado el sector eléctrico desde el siglo 19 pasando por la era del carbón tomando a la aplicación con el objetivo de electrificar a la sociedad, luego en el siglo 20 con una generación extensiva de energía eléctrica con la era de los combustibles fósiles, en el siglo 21 con un concepto de la nueva electricidad que han implicado cambios demográficos, escasez de recursos, cambio climático; estos son antecedentes que permiten imaginar este nuevo modelo o la denominada nueva era de la electricidad, pensando como la fuente de energía para todas las aplicaciones del día a día, obteniendo un sistema energético integrado. En este particular se enmarca una generación centralizada y descentralizada, inteligente con el uso de TIC's y con un flujo de energía bidireccional adicionando fuentes de energía renovables como solar, eólica, hidráulica, biomasa, geotérmica, etc. Este modelo se centra en incrementar las fuentes renovables con gestión de su red, capaz y confiable, para colaborar en la reducción de emisiones, disminuir el empleo de generadoras termoeléctricas, incluir vehículos [13] eléctricos y buscar la eficiencia energética mediante una gestión de la demanda y facilitar la construcción de edificios inteligentes.

Desde las primeras experiencias de implementación de redes eléctricas inteligentes se han buscado obtener una mayor eficiencia del sistema eléctrico, reducir el gasto energético disminuyendo las pérdidas en la distribución, generación de energía en gran parte buscando aportar al medio ambiente y al coste que genera, se han presentado varias alternativas como políticas de cada país, pero sin duda alguna que la inversión económica ha resultado un obstáculo y una proyección de mediano y largo plazo, desde la realidad de Ecuador se ha comenzado con la capacitación en los 
sectores gubernamentales con las empresas de distribución, consejo de regulación, centro de control de energía y los colegios de ingenieros, los primeros pasos dados hasta el momento han sido referentes a analizar diferentes propuestas internacionales como la hoja o mapa de ruta que promueven ciertas particularidades, obviamente desde la propuesta de cada país y de acuerdo a su realidad del sistema eléctrico. Además se ha empezado con la automatización mediante el uso de IED's en las protecciones de las subestaciones, incluyendo como valor agregado la posibilidad de realizar cortes y reconexiones de forma remota. Se ha incluido un sistema GIS georeferencial de cada subestación y sub-etapa de distribución para las ciudades principales, esta es una alternativa propia de cada empresa eléctrica, pues en Ecuador tienen su participación accionaria en representación de los municipios locales de cada ciudad que llegan hasta un $56 \%$ y el resto obedece a control y regulación gubernamental, se ha tomado como línea de base la experiencia en el sistema GIS de una empresa eléctrica y el sistema de facturación de otra empresa, con la finalidad de implementar en el restante grupo de empresas distribuidoras. Es decir es una decisión emergente en busca de crear un sistema estandarizado y homologado por el ente regulatorio, así se podrá obtener una sola base de datos, datos históricos, análisis de cargas, entre otros. En la actualidad en Ecuador se hacen pruebas a nivel de transformadores antes y después, para analizar diferentes tecnologías que colaboren a reducir las pérdidas no técnicas provenientes de usuarios de diferentes situaciones sociales, con este sistema se tendría el valor real de consumo del abonado (doméstico/industrial) sin necesidad de enviar a un personal de lectura de medidores, es decir son pequeñas alternativas ais- ladas que se presentan con trabajo multidisciplinario entre ciertas empresas de distribución y la universidades.

El corte y reconexión del suministro hasta el momento se lo realiza de forma manual y la lectura tiene errores y reclamos de los usuarios por no ser en tiempo real y confiable, este es uno de los temas de importancia ya que se necesita cambiar medidores inteligentes y proveer redes de comunicación para llevar la información desde los diferentes abonados hasta el centro de control y facturación del suministro eléctrico.

Han sido varios los proyectos a nivel nacional, sudamericano, europeo y norteamericano donde las comunicaciones dan el soporte básico para que un Smart Grid pueda ser implementado, pero existen varios inconvenientes en cada país y sobre todo en los que están en inicios de desarrollo como lo es Ecuador, los planteamientos de los diferentes protocolos presentan un abanico de opciones pero sin ningún sustento técnico relacionado o una metodología de gestión sobre este tipo de proyectos que oriente de forma específica su empleo en redes eléctricas inteligentes, en Ecuador se hicieron varias pruebas en BPL/PLT para dotar de servicios de internet con costos sumamente elevados y por políticas del estado se eliminó esta competencia de las empresas distribuidoras, el costo fue elevado debido a que la red actual no tuvo visión de futuro, es aérea, con mucho ruido y propensa a hurtos y manipulación por lo que este protocolo no tuvo éxito.

Dentro de los servicios requeridos se encuentran aplicaciones útiles como:

- Localizar interrupciones en el servicio

- Sistemas automáticos de medidas (remotos)

- Sistemas de control supervisado y adquisi- 
ción de datos (SCADA)

- Fallas en alimentadores de distribución

- Eventos climáticos en las líneas de transmisión

- Entrenamiento de equipo de mantenimiento e inspección de alimentadores

- Localización de fallas en líneas de transmisión

Dentro de los casos reales de estudio está el norteamericano y las iniciativas de Europa.

- Smart Metering: Medición Inteligente

- Smart Cities: Ciudades eficientes e inteligentes (Domótica)

- Grid Intelligence: Gestión Inteligente de Redes y de los Sistemas Eléctricos

A partir de estos antecedentes se pude advertir tres redes de comunicaciones necesarias, donde se pretende fundamentar el estudio y colaborar con la sustentabilidad del modelo smart grid [8], entonces tenemos:

- Home Area Network

- Building Area Network

- Industrial Area Network

Se advierte que con la implantación de una red de distribución activa, permitirá la creación de micro redes, redes de baja tensión con fuentes de generación distribuida, dispositivos locales de almacenamiento y cargas controlables. Las necesidades para gestionar servicios adicionales a los consumidores se basan en la asociación de las redes de comunicación con la etapa AMI, así estos clientes podrán tener una gama de beneficios como el monitoreo del uso de energía, economizar en función del precio de la energía, pero la empresa distribuidora tendrá acceso directo a la carga y así podrán gestionar de forma eficiente la demanda. Entre los avances de uso de redes de comunicación para lograr otros servicios al consumidor tenemos:

WiMax.- Utilizado para la conectividad de la última milla. Censado de la red eléctrica, además puede ser utilizado para interconexión de medidores inteligentes.

3G.- Usado para censado remoto es decir utilizado para control y monitoreo.

MPLS.- Desarrollado para interconexión de redes de diferentes tecnologías.

ZigBee.- Ha estado desarrollando tecnología para comunicación remota entre medidores incluye gas y agua. Está basada en el IEEE estándar 802.15.4, tiene una velocidad de $250 \mathrm{kbps}$, su rango de transmisión es limitado y debe ser combinado con tecnologías de comunicación de mayor rango como las del estándar IEEE 802.11

Home Plug.- Esta tecnología incluye interconexión con bombas de agua, audio, televisión, nevera, iluminación, vehículos eléctricos.

WiFi [14] y 4G LTE.- También son tecnologías aplicadas para comunicaciones inalámbricas pero pueden ser usadas para mejorar cobertura, ancho de banda o velocidad y conseguir así los datos desde cualquier dispositivo electrónico, es decir es usado para medición inteligente. También 4G LTE puede ser usado para control y monitoreo en alto y medio voltaje, conectividad con hogares [15] de sectores urbanos y para sectores rurales se puede implementar WiMax ya que debido al empleo de altas frecuencias en $4 \mathrm{G}$ no es recomendable. En lo referente a BAN (Building Area Network) es posible asociar diferentes tecnologías como son 802.16 WiMAX, 802.11 WiFi, Fibra óptica, 802.15.4/zigbee todo con el interés de dotar aplicaciones satisfactorias como: 
- Seguridad en una red eléctrica inteligente

- Privacidad

- Medición Inteligente de Fraude

- Ataques maliciosos

- Compresión de datos para evitar congestión

Para esto es necesario conseguir la interoperabilidad de las diferentes tecnologías de comunicación con sus respectivas interfaces, dicha capa puede ser IPV6. Toda la tecnología existente puede sin problema abarcar de extremo a extremo con la arquitectura de comunicaciones en una red eléctrica inteligente, las mismas que permitirán dotar de servicios adicionales a los consumidores en conjunto con su metodología de gestión de un proyecto para aplicación a dichas redes.

De acuerdo a estos antecedentes es importante tener en cuenta que la planificación es factor fundamental y a más de tener los documentos de planificación específicos referente a los insumos físicos y financieros, el personal, las cantidades, los resultados físicos esperados, se debe tener un análisis adecuado de los objetivos, grupos financieros y los factores externos que podrán determinar el resultado de un proyecto con éstas características.

Será importante también entonces tener en cuenta las políticas que se relacionan estrechamente al resultado del proyecto ya que buscan responder a las necesidades de los consumidores/beneficiarios propuestos. Entonces se podrá plantear para este proyecto tres fases importantes como: Fase de Inicio y Planificación; Fase de Ejecución y Control; Fase de cierre de Proyecto [16].

En el inicio y la planificación se dispondría del diseño del equipo de gestión del proyecto, la creación del plan de proyecto, orientación y preparación de recursos, aprobación del plan de proyecto. En la ejecución del proyecto está la relación entre la ejecución del plan del proyecto, el seguimiento mismo del proyecto, revisión o control de cambios, reporte de los datos, modificación del proyecto si es pertinente y la etapa aceptación del proyecto. Finalmente dentro del cierre del proyecto estará la evaluación del proyecto con su cierre y transferencia de conocimientos a todas las instancias involucradas, tanto en la etapa de generación como en la etapa final-consumidor.

Las etapas básicas de un Smart grid seguirán siendo importante, es necesario el uso de medidores digitales avanzados, automatización, sistemas de comunicación y recursos energéticos distribuidos que integren varias tecnologías.

El concepto de eficiencia dentro de los sistemas eléctricos deberán evidenciar características como:

\section{Reducción de pérdidas de energía}

- Pérdidas técnicas en la red.

- Pérdidas comerciales por bajas de clientes o no facturación.

\section{Disminuir el coste de operación por red}

- Energía no entregada.

- Elevado número de operaciones de conmutación.

\section{Disminuir el coste por desarrollo de la red}

- Elevada utilización de las instalaciones de distribución.

- Retraso en inversiones.

Mejora de la calidad de servicio 
- Regulación de tensión en la línea con interruptores en servicio.

- Regulación off-line: auto curable y adaptable.

- Resiste ataques y disturbios.

\section{Mejora de Interactividad}

- Integrar datos y funciones.

- Interacción entre el mercado eléctrico y los usuarios.

En el área de la generación se deberán continuar con los avances sobre la base actual, entre ellas tenemos:

- Generación distribuida a gran escala.

- Gestión integral con mayores actores

- Generadores puros

- Generadores virtuales

- Generadores/almacenadores

- Almacenadores puros.

- Nuevas técnicas de almacenamiento

- Aprovechamiento de energía marginal proveniente de autos eléctricos.

En transporte y distribución también podemos proyectar opciones de cambio como:

- Incrementar nuevas tecnologías que permitan incrementar la capacidad de la red, optimizar la topología y reducir las pérdidas entre las que tenemos:

- FACTS (Compensación de potencia reactiva en líneas de transmisión)

- Superconductores
El desarrollo basado en esos dispositivos semiconductores primero estableció la tecnología de transmisión DC a alto voltaje HVDC, como una alternativa para la transmisión $\mathrm{AC}$ a largas distancias, y ésta tecnología, a su vez, estableció la base para el desarrollo de los sistemas de transmisión flexible en AC, FACTS, equipos que pueden ser usados para resolver los problemas en sistemas de transmisión AC. Los controladores FACTS [17] son el resultado de una combinación de equipos convencionales de compensación, tecnología de punta en electrónica de potencia y de microelectrónica. Estos aspectos sumados al uso de los avances en el área de las comunicaciones industriales, hacen de los dispositivos FACTS equipos que contribuyen a una operación óptima de un Sistema Eléctrico de Potencia (SEP).

Así también existen otros sistemas que permiten:

- Incremento en la sensorización de las redes y comunicaciones

- Mantenimiento preventivo: CBM y gestión de vida

- Monitorización de cuellos de botella

- Supervisión compartida de múltiples redes geográficamente superpuestas.

- Sistemas expertos

- Apoyo en la toma de decisiones óptimas.

- Ajuste automático del sistema.

- Gestión de nuevos usos/demanda- Bidireccional.

- Nuevas arquitecturas de red eléctrica que permita obtener los beneficios asociados a las nuevas tecnologías. 


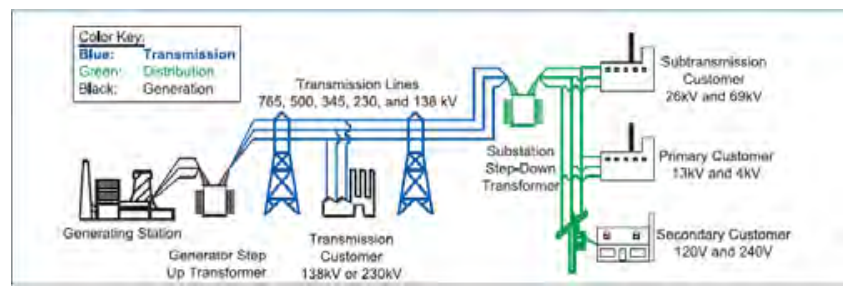

Figura 2. Estructura básica del sistema eléctrico actual (Canadá) [18]

Para llegar a una red eléctrica inteligente deberemos tomar en cuenta ciertos parámetros que en la actualidad ya tienen mucha penetración en el mercado eléctrico como:

Telemedida Lectura de la energía de manera remota.

- Contador, elemento pasivo de comunicación unidireccional.

- Pocas necesidades de comunicación.

Telegestión (SmartMeter) Implica una revolución del equipo de medida y por supuesto de la red.

- Contador puede ser leído y gestionado de manera remota.

- Altas necesidades de comunicación bidireccional.

\subsection{Criterios para implementar una red eléctrica inteligente}

En los actuales momentos son muchos los planteamientos para acceder a la interdisciplinaridad de las tecnologías pero sobre todo romper las barreras.

En esto se denota tres articulaciones: Operaciones Inteligentes, Medición Inteligente y Redes Inteligentes, los mismos que se integran mediante un proceso de negocio. Además se debe tener en cuenta aspectos económicos como:
- Madurez tecnológica y riesgo de "first mover".

- La falta de tecnologías estándares y maduras, aumentan el riesgo de inversión, además están las escasas pruebas piloto de escala suficiente que no permiten que las estimaciones y supuestos considerados no sean totalmente fiables.

- "Business case"

- Los costes de inversión y operación son todavía elevados y por otra parte los beneficios que se pretenden conseguir son difícilmente cuantificables.

- Concienciación.

- De parte de los reguladores enmarcados en los objetivos de fomento de las energías renovables, eficiencia energética, reducción de $\mathrm{CO}_{2}$ y la necesidad de aumentar la inversión en las redes eléctricas.

- Normativa de regulación.

- En algunos casos impone limitaciones o barreras técnicas y en otras no genera incentivos suficientes para la inversión.

- Acceso a fuentes de financiación.

- Con cambios repentinos en el modelo y el aumento de los riesgos de una actividad regulada permiten el aumento de costes de financiación haciendo menos rentables las inversiones.

\subsection{El cambio cultural de empresas eléc- ticas y consumidores}

Entre las proyecciones de las empresas eléctricas deberán existir las interrogantes que en 
una red eléctrica tan grande e inteligente debe haber un constructor, es decir un diseño del proyecto, supervisión y posterior construcción. En estudios realizados se han demostrado que al desconectar los equipos de las fuentes de poder se pueden ahorrar un significativo valor en corriente y por ende en sus facturas mensuales, pueden existir reducciones entre $5 \%$ y $15 \%$ por cada hogar. Se podría deducir entonces que la causa para los grandes consumos de energía se relaciona directamente a la tecnología y sus posibles soluciones para ahorro energético.

El Smart Metering ingresa entonces en ésta planificación inteligente, permitiendo a los hogares supervisar el uso de la energía enviando en tiempo real datos a sus proveedores de energía, su inversión será costosa por la tecnología necesaria en su desarrollo. Las tecnologías de energía inteligente pueden ofrecer beneficios excepcionales, permitiendo comunicar a los proveedores y los clientes, abriendo el camino para ajustar el consumo a las condiciones y los precios en tiempo real. Esta respuesta a la demanda contra taca con el sistema tradicional en el que el suministro debe cubrir la demanda.

\section{Proyectos generados en smart grid}

\section{INTEGRIS}

El Proyecto INTEGRIS, INTelligent Electrical GRId Sensor communications (Comunicaciones de Sensores para Redes Eléctricas Inteligentes), que tiene por objetivo diseñar y desarrollar una infraestructura de telecomunicaciones robusta y flexible, capaz de obtener y canalizar de forma eficiente y en tiem- po real toda la información que requerirán las nuevas redes inteligentes de distribución eléctrica. Los proyectos orientados en ésta línea, permitirá la "óptima gestión" de la oferta y la demanda, así como la minimización de las pérdidas, la mejora de la gestión de activos y hacer más sencilla la operación de la red.

Las redes eléctricas, las redes de sensores y las redes de comunicaciones tienen más puntos en común de lo que se cree. La parte de comunicaciones del proyecto INTEGRIS se centra en las SmartGrids y tiene por objetivo especificar una plataforma integrada para la comunicación con independencia de la tecnología utilizada. "Las tecnologías en las que se centra este proyecto son: Power Line Communications (PLC), Wireless [20] Sensor Networks (WSN) y Radio Frequency IDentifiers (RFID)". Esta plataforma proporcionará redundancia a la red de comunicaciones PLC de una red eléctrica a través de WSN, a la vez que aporta acceso a los sensores, todo ello íntimamente relacionado con las SmartGrids. Otro objetivo de esta plataforma es facilitar la gestión de la red, sin olvidar términos tan importantes para la telemática como son: la QoS, el routing o la seguridad. Los integrantes del consorcio son: Endesa, La Salle, TUT,

Schneider-electric, CTI, ILIGHT, DS2, A2A e INDRA.

\section{EPRI IntelliGrid [21]}

Tiene como objetivo crear una infraestructura eléctrica de entrega de potencia en forma interdisciplinaria junto a las comunicaciones, informática [22] y electrónica y así satisfacer las necesidades de energía de los años venideros y facilitar la transformación de la infraestructura eléctrica proporcionando una mayor seguridad, alta calidad, confiabilidad. Su proyec- 


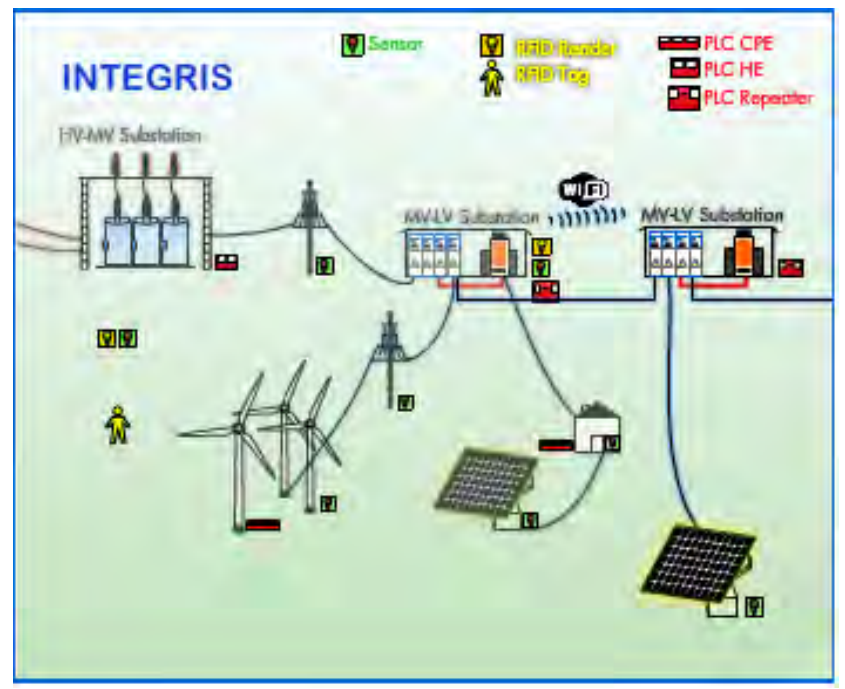

Figura 3. Proyecto planteado por Integris. [19]

to ADA se caracteriza por tener sistemas de distribución automatizados mediantes sistemas flexibles operados a través de una arquitectura de sistemas de control y comunicación. Este sistema mantiene los puntos específicos en mejora de la fiabilidad y la calidad de la energía, reducción de costes por funcionamiento, reducción del tiempo de restauración luego de un corte, aumento de opciones de servicio para el cliente, integración de la generación distribuida, opciones de almacenamiento e integración de los clientes al sistema.

\section{GridWise Alliance}

Proyecto lanzado por el Departamento de Energía DOE [24] US en alianza con EPRI - Intelligrid y Galvin Electricity Initiative, el mismo está centrado en una red moderna en infraestructura en la distribución y sus operaciones que utiliza dos vías de flujo de electricidad y de información.

En la actualidad empresas como GE se encargan de fabricar hardware y software para smart meters (medidores inteligentes), empre- sa que ha ganado varios contratos con grandes servicios. Empresas PG\&E instala 3.3 millones de medidores inteligentes de GE en California y American Electric Power plantea instalar inicialmente 200.000 medidores inteligentes con una proyección de 5 millones de usuarios para el año 2015.

Xcel estuvo implementando una versión limitada del experimento con el que busca, entre otras cosas, evaluar la reacción de los usuarios, medir la conservación de energía y cuantificar los ahorros de costos. Ha equipando unos 10.000 hogares con aparatos gratuitos que pueden controlar ciertos electrodomésticos de manera remota. Los clientes pueden visitar el portal de Internet de la empresa y establecer metas para el uso de energía, el usuario puede cancelar sus preferencias si así lo desea.

\section{Telegestore (ENEL, ITA 200-2005)}

"Telegestore oferta una lectura remota del consumo, estructura multi-tarifa, desconexión remota, monitoreo de la calidad del servicio, detección de fraudes, medición, transmisión de datos, concentradores de información en cada 


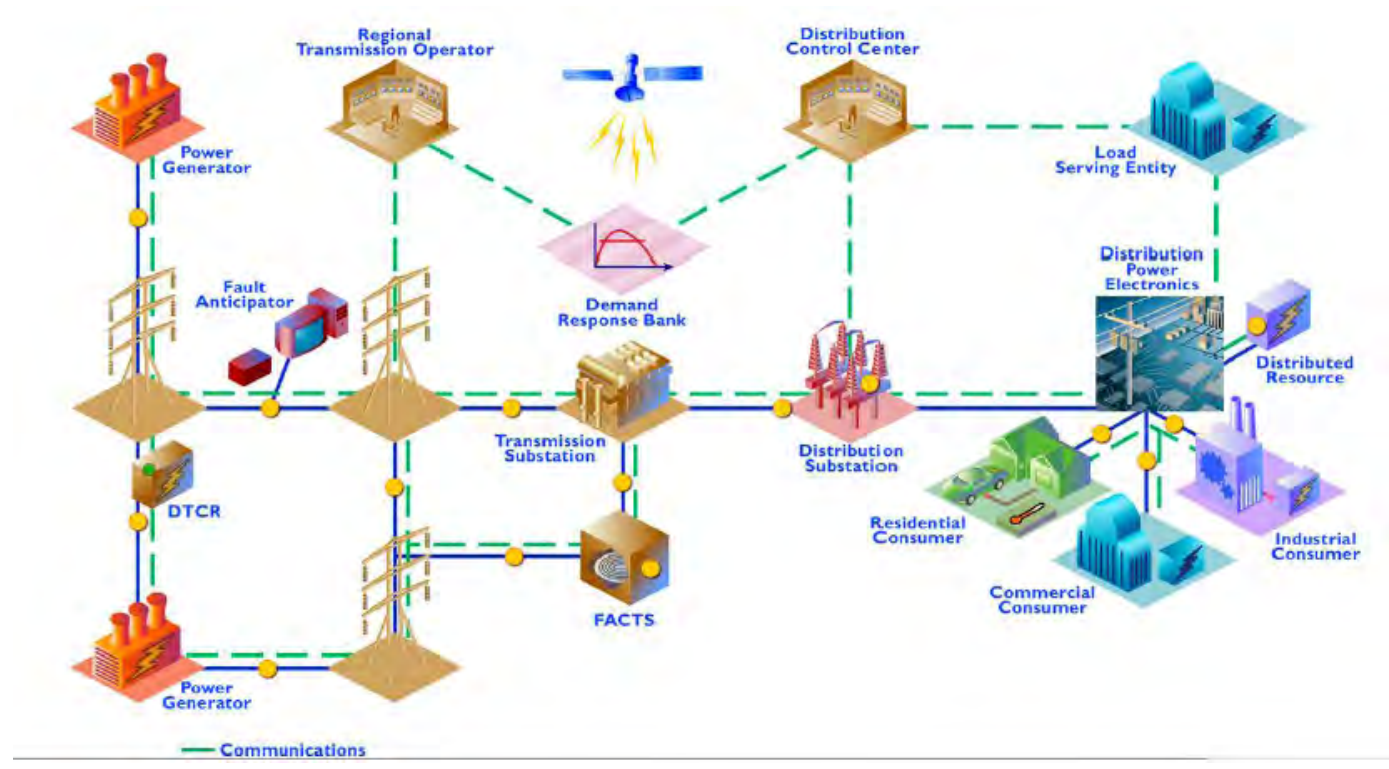

Figura 4. Proyecto Epri IntelliGrid.[23]

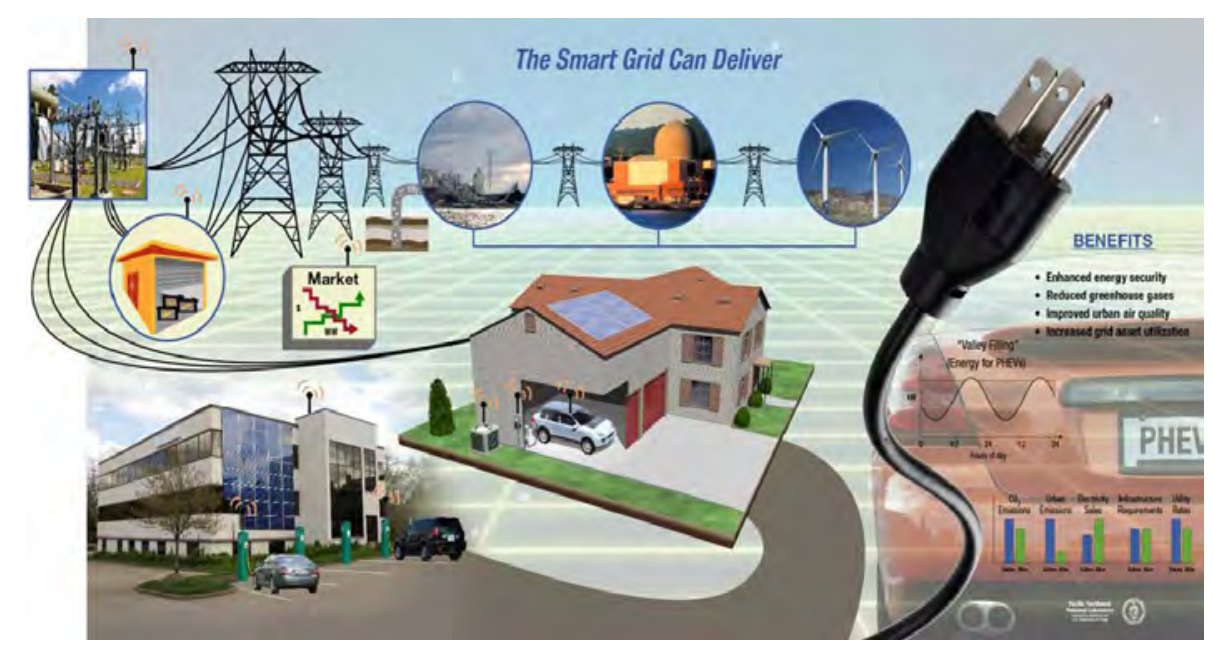

Figura 5. Proyecto GridWise Alliance + DOE. [25]

subestación de distribución, sistema centralizado para administración del proceso".

\section{AVANTI (Southern California - Edison 2009 - 2012) [26]}

Esta solución permite en su concepción ofertar una tecnología avanzada en circuitos de distribución, medidores inteligentes, una infraestructura para recarga de vehículos eléctricos, automatización en distribución, procesadores lógicos, interruptores en vacío y restau- radores automáticos para reducir el tiempo para localizar y aislar fallas, redes de fibra óptica para medición de temperatura en cables y permitir optimización de su capacidad de conducción, utiliza tecnología de FACTS para control flexible del suministro de potencia reactiva. Además posee proyectos demostrativos para incursionar en micro-redes en Bronsbergen (Holanda) y trabaja en la búsqueda de garantizar el suministro y calidad de energía eléctrica para identificar problemas en la integración de micro-redes con el sistema de dis- 
tribución. Su capacidad instalada advierte

- 108 casas con paneles solares

- Bancos de baterías como almacenamiento de energía para demanda pico.

- Centro de despacho y equipo de medición para control del intercambio de energía entre la micro-red y la red de distribución.

\section{Desafíos de smart grid}

Dentro de diferentes líneas de investigación en redes eléctricas inteligentes podemos encontrar puntos en común que permitirán advertir ciertos desafíos que se deben aterrizar hasta la realidad ecuatoriana entre ellos están:

- Asegurar que exista capacidad de transmisión para interconectar las fuentes de energía, especialmente las renovables al mismo tiempo que el impulso para su implementación.

- Dotar de servicios pos-venta dirigido a los clientes.

- Desarrollar conexiones eficientes para el máximo aprovechamiento de energía solar, eólica, geotérmica, etc.

- Desarrollar arquitecturas descentralizadas habilitando sistemas de suministros de energía de menor magnitud con el fin de que operen con facilidad junto al resto del sistema.

- Crear una infraestructura de comunicación para lograr la operación de toda el área y su manejo dinámico en el mercado eléctrico.

- Permitir que los consumidores participen activamente en la operación del sistema.
- Permitir la generación, demanda y uso de energía inteligente.

- Utilizar beneficios de almacenamiento de energía.

- Preparar el camino para el uso de vehículos eléctricos, prestando todas las comodidades a los consumidores. (subsidios e instalaciones residenciales)

- Asegurar el correcto uso de las centrales de generación.

- Considerar las limitaciones del sistema teniendo en cuenta el impacto ambiental.

- Proponer mejoras políticas y regulatorias sobre redes eléctricas inteligentes.

La inversión entonces pasa a tomar un punto crítico para su aplicación, debido a que la tecnología podría ser obsoleta entre 5 y 7 años y los costes estarían proyectados para una recuperación aproximada de 30 años.

Este análisis financiero se podría contrastar con la eficiencia del sistema y si este significa ahorro. El costo por interrupción es considerable en el usuario final referido a costos (comerciales e industriales). En la actualidad la empresa eléctrica dispone en sus inicios de automatización de las subestaciones colocando IED's proporcionando beneficios tales como:

- Mejora de la calidad del servicio y la relación con el cliente, respondiendo a las interrupciones con prontitud.

- Aumentan la capacidad del operador para supervisar y controlar el sistema de alimentación, en condiciones normales, anormales y de emergencia, proporcionando los datos en tiempo real, confiable y adecuado. 
- Aumenta la eficiencia del sistema de alimentación, reduciendo las pérdidas.

- Ayuda a los equipos de mantenimiento y protección, ofreciendo registros más fiables, significativo y oportuno de los históricos de funcionamiento.

- Mejora el análisis y planificación del sistema de alimentación, proporcionando un mayor acceso a los datos de las operaciones pasadas presentes y sus aplicaciones.

\section{Referencias}

[1] A. Estrada, "Tecnologías de Internet para diseñar la red eléctrica del Futuro," Revista Ingenierías USBMed, vol. 2, no. 2, 2011. [Online]. Disponible en: web.usbmed.edu. co/usbmed/fing/v2n2/v2n2.pdf

[2] M. B. and G. M., "Ahorro energético y Telecomunicaciones, Convergencia con la Red Eléctrica y Desarrollo Sostenible," LM Data Communications S.A. Spain, no. 1, Marzo - 2007. [Online]. Disponible en: http://www.lmdata.es/ uets/mma/ethf-mma-ebook.pdf

[3] G. Clark, "The smart grid, enabling energy efficiency and demand response," The Fairmont Press Inc, CRC Press Taylor y Francis group. Lilburn, Georgia USA., vol. 10, p. 300, 2009. [Online]. Disponible en: http://www.fer.unizg.hr/_download/ repository/The_smart_grid-_ enabling_energy_efficiency_and_ demand_response,_CRC_2009-.pdf

[4] Y. Peizhong, I. A., and Z. Chi, "Building automation Networks for Smart Grids," Electrical and Computer Engineering
Deparment. [Volume 2011], vol. 2011, Novemeber - 2010. [Online]. Disponible en: http://www.hindawi.com/journals/ ijdmb/2011/926363/

[5] A. Molderink, "Domestic energy management methodology for optimizing efficiency in Smart Grids," 2009, p. 2009. [Online]. Disponible en: http:// doc.utwente.nl/68163/1/powertech.pdf

[6] A. Borrero, "Tic/ tic vs eficiencia e sustentabilidad energética," IV encontro ibero-americano sobre os objectivos do milénio, 3 e 4 de novembro de 2009.

[7] Spark Magazine, October 2009. [Online]. Disponible en: http://www.themcdonnellgroup.com/ PDF/SPARK_1009.pdf

[8] Issuesletter, "Red Eléctrica Inteligente o Políticas Inteligentes: Qué viene primero?" [Online]. Disponible en: http://aeace08rei.files.wordpress.com/ 2011/05/rap_issueslettersmartgrid_ spanish_2009_07_16.pdf

[9] J. Phitart, "Integration and Utilization of Smart Grid," 2009. [Online]. Disponible en: http://www.feec.vutbr.cz/EEICT/2009/ sbornik/03-Doktorske\%20projekty/ 04-Silnoprouda\%20elektrotechnika\% 20a\%20elektroenergetika/09-xpitha01. pdf

[10] P. Fox-Penner, "Smart Power, Climate change, the smart grid and the future of electric utilities," IslandPress, 2010.

[11] L. M., "End-toEnd Communication Architecture for Smart Grids," IEEE Transactions on Industrial Electronics, vol. 58, no. 4, April - 2011. [Online]. Disponible 
en: http://ieeexplore.ieee.org/xpls/abs all.jsp?arnumber $=5559470$

[12] D. of Energy, The Smart Grid and Indroduction, 2007. [Online]. Disponible en: http://energy.gov/sites/prod/files/ oeprod/DocumentsandMedia/DOE_ SG_Book_Single_Pages\%281\%29.pdf

[13] J. K. Zheng, L. Huang, G. Li, H. Cao, W. Wang, and M. Dohler, "Beyond 3G Evolution," IEEE Vehicular Technology Magazine, vol. 2008, pp. 30 - 36, 2008.

[14] J. Boal, "Smart grid," pp. 2009 - 2010, 2009. [Online]. Disponible en: http://www.dea.icai.upco.es/sadot/ Comunicaciones/avanzadas/Smart\% 20grid\%20-\%20Jaime\%20Boal.pdf

[15] T. Flick and J. Morhouse, Securing the Smart Grid, Next Generation Power Grid Security.1A., 2011.

[16] "Metodología básica de gestión de proyectos," 2003. [Online]. Disponible en: http://www.pcmanagement. es/editorial/Managem_powpoin/ MetodologiadeGestiondeProyectos.pdf

[17] D. Miranda, "Estado y desarrollo de la tecnología smart grid en Colombia." [Online]. Disponible en: http://www.bdigital.unal.edu.co/ 864/1/98699867_2009.pdf

[18] "Blackout in the United States and Canada: Causes and Recommendations," Tech. Rep., August 14, 2003. [Online]. Disponible en: http: //fp7integris.eu/index.php

[19] "INTEGRIS: INTelligent Electrical Grid Sensor communications." [Online]. Disponible en: http://fp7integris. eu/index.php
[20] A. Clark and C. Pavlovski, "Wireless Network for the Smart Energy Grid Application Aware Networks," vol. 2, pp. 1243 - 1248, March 2010. [Online]. Disponible en: http://www.iaeng.org/ publication/IMECS2010/IMECS2010 pp1243-1248.pdf

[21] S. Mark and S. Fred, "Smart Grid. 1A. [2009]," Alejandria Virginia USA: CapitolNet., 2009.

[22] C. Lopez, "Teoría y aplicación de la informática 2," Agosto 2009. [Online]. Disponible en: http://www.jeuazarru. com/docs/SmartGrid.pdf

[23] J. Caskey, "Smart Grid Overview," October 30, 2008. [Online]. Disponible en: www.nema.org/gov/energy/smartgrid/.. ./Smart_Grid_Overview.ppt

[24] A. Molderink, "Simulating the effect on the energy efficiency of smart grid technologies." [Online]. Disponible en: http://www.fer.unizg.hr/ _download/repository/144.pdf

[25] K. Hamilton, "What I Learned in Two Years of Running GridWise Alliance," greentechmedia.com, January 14, 2011. [Online]. Disponible en: http: //www.greentechmedia.com/articles/ $\mathrm{read} / \mathrm{what}$-i-learned-two-years-r\% unning-gridwise-alliance/

[26] H. Sarmiento, "Technical chat "smart grid"," 2010. [Online]. Disponible en: http://www. utpl.edu.ec/blogjorgeluisjaramillo/ wp-content/uploads/2010/10/ IEEE-Day-Smart-Grid-v2.pdf 